\title{
Effects of a vibrational proprioceptive stimulation on recovery phase after maximal incremental cycle test
}

Francesco Coscia (1,2), Paola V. Gigliotti (1,2), Saadsaoud Foued (3), Alexander Piratinskij (4), Tiziana Pietrangelo (5), Vittore Verratti (6), Igor Diemberger (7), Giorgio Fanò-Illic $(8,9,10)$

(1) Department of Medicine, University of Perugia, Ospedale "Santa Maria della Misericordia”, Italy; (2) Laboratory of Sport Physiology, San Candido-Innichen, Italy; (3) Maitre de Conference Class A, Universite de Msila, Algerie; 4) The Ural Federal University, Ekaterinburg, Russia; (5) Department of Neuroscience Imaging and Clinical Science, Interuniversity Institute of Myology, University G d'Annunzio Chieti-Pescara, Italy; (6) Department of Psychological Sciences, Health and Territory, University “G. d'Annunzio" of Chieti-Pescara, Chieti, Italy; (7) Institute of Cardiology, University of Bologna, Azienda Ospedaliera S. Orsola-Malpighi, Italy; (8) IIM-Interuniversity Institute of Myology; (9) A\&C M-C Foundation for Translational Myology, Padova; Italy; (10) Free University of Alcatraz, Santa Cristina di Gubbio, Italy.

This article is distributed under the terms of the Creative Commons Attribution Noncommercial License (CC BY-NC 4.0) which permits any noncommercial use, distribution, and reproduction in any medium, provided the original author(s) and source are credited.

\begin{abstract}
Global Proprioceptive Resonance (GPR) is a recently developed approach conceived to solicit the various cutaneous mechanoreceptors, through application of mechanical multifocal vibration at low amplitude and at definite body sites, limiting the stimulation of the profound structures. This interventional study evaluated the effects of GPR on cardiorespiratory function during the post-exertional recovery period. A group of volunteers involved in Triathlon (a multisport discipline consisting of sequential swim, cycle, and run disciplines higly demanding in terms of metabolic engagment), underwent two maximal incremental exercise tests until exhaustion followed alternatively to (a) a 13 minutes section of GPR or (b) a standard low intensity exercise acute trend of the same duration. These effects of these two approaches were compared in terms of recovery of: heart rate (HR), respiratory rate (RR), peripheral oxygen saturation (SpO2) and venous lactate concentration (Lac). The physiological parameters (HR, RR, SpO2 and Lac) recorded in the pre-exertion session showed similar values between the 40 volunteers while several differences were recorded in the post-exertion phase. After 6 min of GPR recovery it was recorded a drop in RR below baseline $(19.4 \pm 4.15 \mathrm{~min}-1$ vs. $12.2 \pm 0.4 \mathrm{~min}-1 ; \mathrm{p}<0.001)$ coupled with an increase in peripheral oxygen saturation above the baseline (GPR: $99.0 \% \pm 0.16 \%$ vs. $96.6 \% \pm 0.77 \%, \mathrm{p}<0.001$ ). Moreover, the most striking result was the drop in lactate concentration measured after 13 min of GPR recovery: $84.5 \pm 3.5 \%$ in GPR vs $2.9 \pm 7.6 \%$ reduction in standard recovery $(\mathrm{p}<0.001)$. Notably no differences were recorded recovery of heart rate. GPR has promising effects on post-exercise recovery on RR, SpO2 and lactate level on young athletes.
\end{abstract}

Key Words: global proprioceptive resonance, recovery phase, incremental test.

Eur J Transl Myol 30 (4): 9477, 2020. doi10.4081/ejtm.2020.9477

Proprioceptive signals from mechanoreceptors of the joints, muscles, tendons, and skin are essential for the intact neural control of movement. The loss of proprioceptive afferents may affect the control of muscle tone, disrupts postural reflexes and severely impairs spatial as well as temporal aspects of voluntary movement. ${ }^{1,2}$ Given the importance of proprioception, numerous interventions have been developed to serve as proprioceptive-training to improve/restore motor function and proprioception. Global proprioception specifically deserves attention in the term that it could influence the skeletal muscle resistance to movement. In particular, the resistance manifests itself as a substantial stiffness for small movements. This aspect could be fundamental in performance overall in highly demanding sport like Triathlon. In fact, the triathletes are involved in 
endurance based-training and in competitions that require to taking advantage from several factors as the economy of locomotion as well as physiological characteristics and also from proprioceptive response. ${ }^{3}$ Notably, there is little agreement of what actually constitutes proprioceptive training, which may be partially owed to the lack of a uniform definition of proprioception. ${ }^{4}$ Broadly defined, proprioception refers to the conscious awareness of body and limbs and has several distinct properties: passive motion sense, active motion sense, limb position sense, and the sense of heaviness. However, it has long been established that proprioception has an unconscious component in which proprioceptive signals are used for the reflexive control of muscle tone and of the posture. ${ }^{5}$ However, proprioceptive signals are also integrated in the complex systems for regulation of cardiorespiratory function, to adapt the body to physical activity. Whole body vibration (WBV), based on somatosensory stimulation, is one of the approaches to global proprioceptive stimulation that has been more extensively investigated, with conflicting result. Recently, mechanical multifocal vibration at specific frequencies and of short duration, has been suggested to efficiently work as Global Proprioceptive Resonance (GPR), with positive effect on bone, muscle and joints. ${ }^{6}$ The aim of this prospective interventional study was to assess whether a new approach to proprioceptive stimulation, named GPR, has positive effects on central or peripheral cardiovascular system on young Triathletes after exhausting exercise engagement during specific test session. ${ }^{7}$

\section{Materials and Methods}

\section{Subjects}

This study was carried out in accordance with the principles outlined in the Declaration of Helsinki. All participants provided written informed consent. Forty athletes involved in long-lasting competitions (triathlon) volunteered for the study. They all were with no recent history of illness or clinical musculoskeletal disorders or contraindications to vibration exposure. The present study was approved by the institutional ethics committee on human research.

\section{Study design}

The study description was reported in Figure 1. The volunteers were randomly divided in two groups (Control and GPR). Participants completed both trials in a quiet temperature-controlled room $\left(23{ }^{\circ} \mathrm{C}\right)$ at the same time period to eliminate any influence of circadian variation. The subjects visited the laboratory on the occasions, with at least 48 hours apart from each occasion: (a) a familiarization of laboratory-equipment, (b) an exercise test to exhaustion followed by (c1) an active recovery period using GPR stimulation or (c2) or an active recovery without GPR stimulation (noGPR). Specifically, all the volunteers were engaged in the exhaustive incremental test at then splitted in two sub-
Fig 1. Scheme depicting the entire study protocol

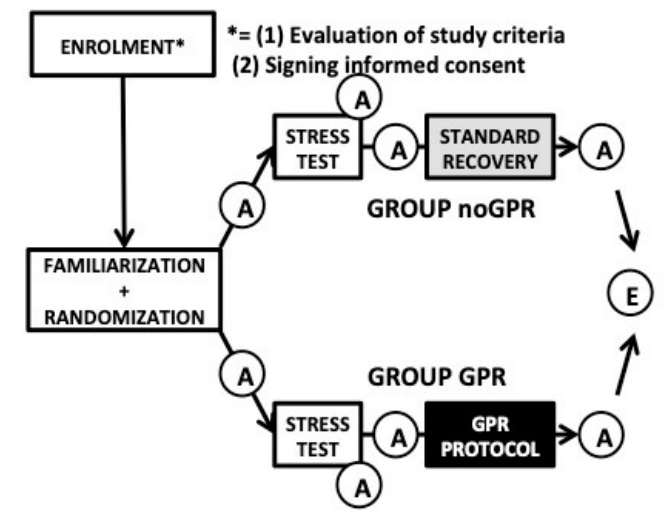

(A) = measure $\mathrm{HR}, \mathrm{RR}, \mathrm{SpO}_{2}$, Lactate (E) = End, data analysis

$G P R=$ global proprioceptive resonance, $H R=$ heart rate, $R R=$ respiratory rate, $\mathrm{SpO}_{2}$ peripheral $\mathrm{O}_{2}$ saturation, Lactate = lactate concentration.

groups of 20 volunteers, one group completed GPR recovery and the other one completing low intensity recovery. Subjects were asked to abstain from exercise for $24 \mathrm{~h}$ and to fast at least $4 \mathrm{~h}$ prior to laboratory visits to reduce the effects of these factors on the study variables.

\section{Incremental exercise test}

After a brief baseline evaluation at rest with 12 lead ECG, blood pressure measurement, peripheral oxygen saturation and peripheral blood lactate assessment, subjects were asked to perform a brief warm-up (3 min, $25 \mathrm{~W}, 60 \mathrm{rev} \mathrm{min}-1)$ on a braked cycle ergometer (Kettler Axiom P2, GmbH \& Co.KG, Ense-Parsit, Germany) Cicloergometro Monark mod 818) prior to the incremental exercise test. The cycling exercise began at an initial workload of 50 watts at a pedaling rate of $60 \mathrm{rev}$ min-1, followed by increments of $25 \mathrm{~W}$ every 2 min until exhaustion. Maximal effort was confirmed by attainment of at least three criteria: (1) a respiratory exchange ratio (RER) $>1.2$; (2) heart rate $>90 \%$ of age-predicted maximum; or (3) rating of perceived exertion $>17$ on the Borg's 6-20 scale.

\section{Recovery period}

After the incremental exercise test, all participants rested in the seating position for two minutes followed by a randomly assigned 13-min recovery periods with: (1) low intensity exercise recovery on the same cycle ergometer (fixed 25 watts) and no GPR stimulation (no GPR) or (2) GPR with no exercise. This recovery period was applied since as previous studies reported, complete muscle recovery can be achieved with $10-15$ min rest periods. We used low intensity exercise recovery to avoid overestimation of the effects of GPR. ${ }^{8}$ 
Vibrational proprioceptive stimulation

Eur J Transl Myol 30 (4): 9477, 2020. doi10.4081/ejtm.2020.9477

\begin{tabular}{cccccc} 
& \multicolumn{5}{c}{ Table 1. Characteristics of Triathlon athletes } \\
Populations & Age & Weight $(\mathbf{k g})$ & Height $(\mathbf{c m})$ & BMI & SBP (mmHg) \\
\hline Control & $22.1 \pm 3.33$ & $73.3 \pm 4.82$ & $182 \pm 4.93$ & $22.3 \pm 2.27$ & $111 \pm 7.59$ \\
\hline GPR & $22.9 \pm 3.28$ & $73.7 \pm 4.67$ & $182 \pm 5.11$ & $22.4 \pm 2.31$ & $111 \pm 7.59$ \\
\hline
\end{tabular}

BMI: body mass index; SBP: sistolic blood pressure;Shapiro-Wilk test for the normality of the distributions; Levene's test for the equality of variances; Repeated Measures ANOVA with BMI as covariate; in addition Tukey correction for multiple post-hoc comparison; when required from Mauchly's test for sphericity, it was applied the Greenhouse-Geisser correction

\section{GPR protocol}

The exercise protocol during GPR matched the standard protocol until the second minute after peak exercise. The subject then was positioned on the Keope GPR system with activation of the stimulation protocol. At the end of the stimulation (lasting 13 minutes, the same as active recovery phase) the subject underwent the last assessment of lactate concentration and the study session ended.

\section{Keope GPR system}

Keope GPR (ANDROMEDA S.r.l. 23896 Sirtori (LC, Italy) is a recently marketed device specifically developed for stimulation of the proprioceptive system by application of low amplitude mechanic forces at specific body sites: hands ankles and heels. The amount of kinetic energy is controlled to limit the stimulation of the profound structures (tendons, joints and viscera) while soliciting the various cutaneous mechanoreceptors: Meissner corpuscles, Ruffini endings, Merkel discs and Pacinian corpuscles. The energy is delivered through sinusoidal wave trains modulated both in terms of amplitude and frequency (until $200 \mathrm{~Hz}$ ). Several stimulation programs have been developed to obtain different patterns of proprioceptive stimulation. Notably, the five stimulation sites are not contemporary activated during all the protocol but they varies within each protocol and between different stimulation programs. A further element that specifically characterizes the Keope GPR system is the position maintained by the subject during the stimulation protocol. Differently from several devices for body mechanical stimulation which require a standing position, the subject performing a section of Keope GPR lies in a relaxed supine position transferring all the gravitational energy through the six stimulating parts and four additional sustaining structures. Notably, the specific shape adopted for all the components of Keope allow a relaxed position without compression of the vascular and lymphatic structures to favour circulation.

\section{Lactate assessment}

The assessment of lactate concentration was performed at: (1) baseline, (2) after reaching the peak exercise, (3) after the first two minutes rest and (4) after the 13 minutes recovery period both with active exercise GPR and with

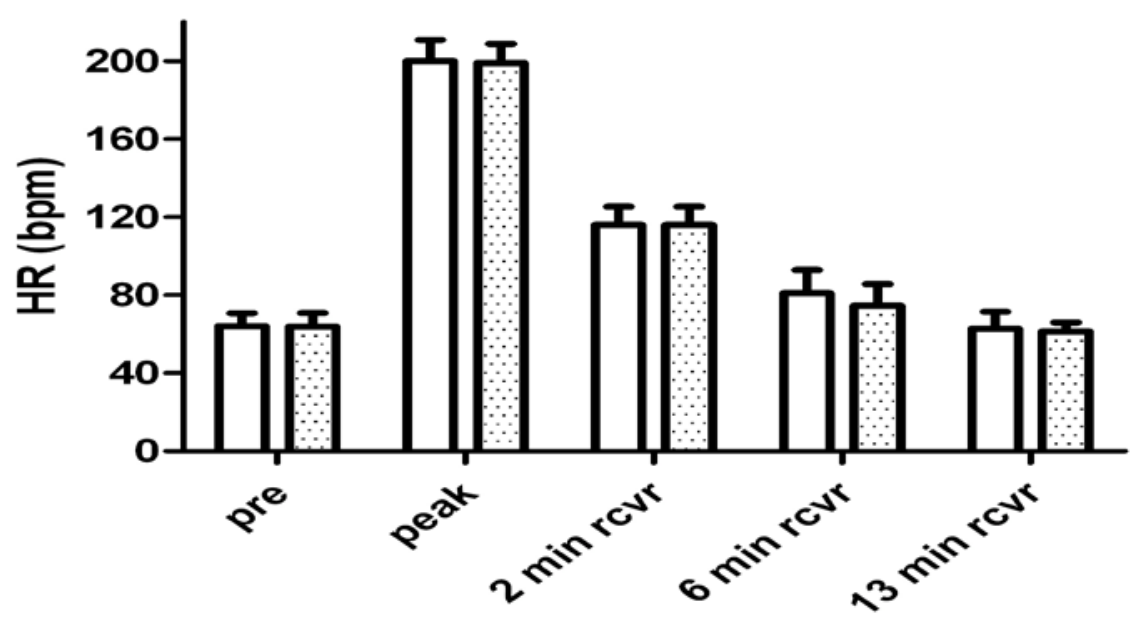

Fig 2. The graph shows the heart rate recorded in all the sessions: before (pre) of the exhaustive exercise, at its maximal level (peak) and during the recovery at 2, 6 and 13 minutes (2 min rcvr, 6 min rcvr and 6 min rcvr). The empty bars represent control group while dotted ones the GPR group. 


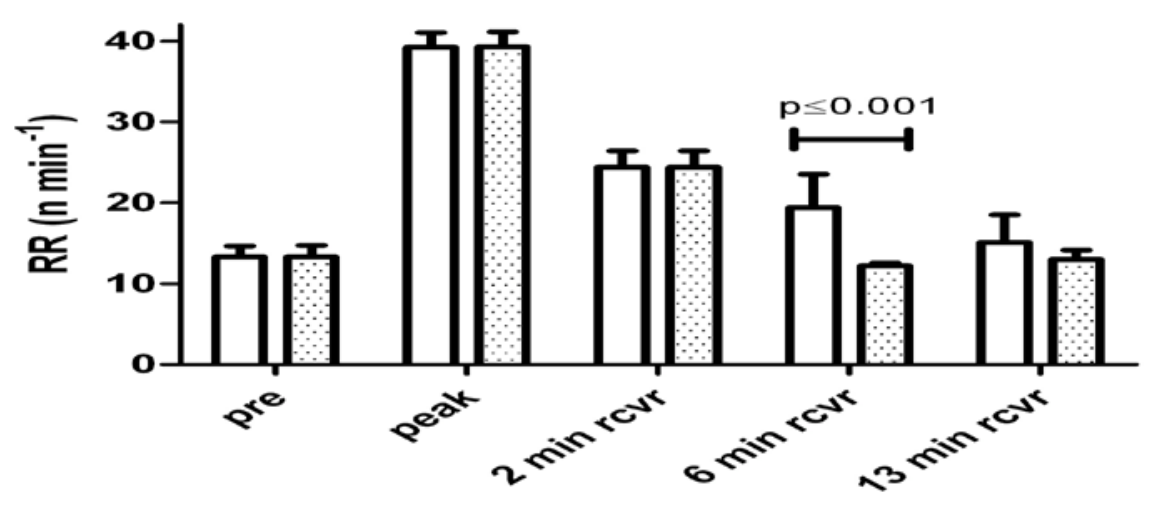

Fig 3. The graph shows the respiratory rate $(R R)$ recorded in all the sessions: before (pre) of the exhaustive exercise, at its maximal level (peak) and during the recovery at 2, 6 and 13 minutes (2 min rcvr, 6 min rcvr and 6 min rcvr). At 6 min of recovery we observed a significant decrease of the RR in the GPR group with respect to control $(p \leq 0.001)$. The empty bars represent control group while dotted ones the GPR group.

exercise noGPR. The blood was collected through a puncture of the distal phalanx of the ring finger of the nondominant hand. Before the collection, the puncture site was aseptically cleaned with $70 \%$ alcohol. The first drop of blood was discarded, then a sample of $30 \mu \mathrm{L}$ of blood was collected, which was analyzed using a lactate monitor Accutrend Plus (Roche Diagnostics) validated by Perez et al., ${ }^{9,10}$

\section{Statistical analysis}

The statistical analyses were carried out using GraphPad Prism Software, version 7 (GraphPad Software, La Jolla, USA), and R-based open-source softwares Jamovi (https://www.jamovi.org). Shapiro-Wilk test for the normality of the distributions and Levene's test for the equality of variances were used. The Repeated Measures ANOVA with BMI as covariate was used to assess the significance; in addition Tukey correction for multiple post-hoc comparison was used; when required by Mauchly's test for sphericity, it was applied the Greenhouse-Geisser correction. The statistical significance was set at $\mathrm{p}$ values less than 0.05 .

\section{Results}

Population and incremental exercise tests

The forty adults volunteers (21 males and 19 females, height $182 \pm 4.9 \mathrm{~cm}$, weight $73.5 \pm 4.7 \mathrm{Kg}$ ) aged $22.5 \pm 3.3$ years, completed all procedures. The subject characteristics of athletes randomly selected for control and experimental group were reported in Table 1 . Notably, no significant difference was recorded among the two different exercise sections in terms of workload (225.85 \pm 18.2 watt vs. $225.75 \pm 18.0$ watt; $p=n . s$.$) and$

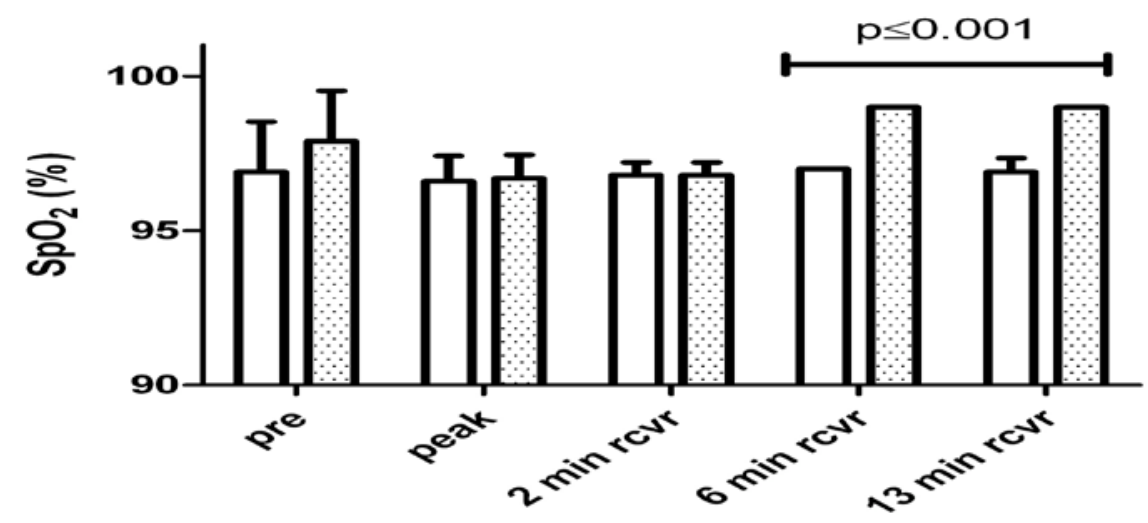

Fig 4. The graph shows the peripheral blood oxygen saturation (SpO2) recorded in all the sessions: before (pre) of the exhaustive exercise, at its maximal level (peak) and during the recovery at 2, 6 and 13 minutes (2 min rcvr, 6 min rcvr and 6 min rcvr). At 6 and 13 min of recovery we observed a significant decrease of the parameter in the GPR group with respect to control ( $p \leq 0.001$ for both points). The empty bars represent control group while dotted ones the GPR group. 


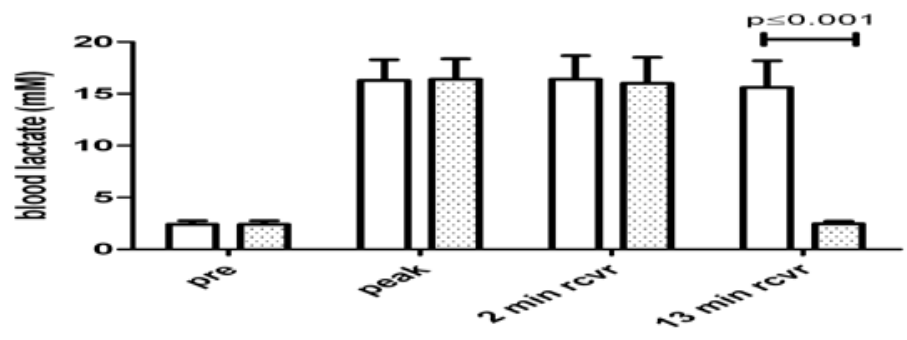

Fig 5. The graph shows the peripheral blood concentration of lactate recorded during the experimental protocol: before (pre) of the exhaustive exercise, at its maximal level (peak) and during the recovery. At 13 min of recovery we observed a significant decrease of the lactate concentration in the GPR group with respect to control ( $p \leq 0.001)$. The empty bars represent control group while dotted ones the GPR group.

physiological modifications (i.e. blood pressure, peripheral oxygen saturation, respiratory frequency, lactate.

\section{Post exercise recovery phase: GPR vs. standard recovery}

Figures 2, 3, 4 and 5 show the modifications of heart rate, respiratory frequency and peripheral oxygen saturation and lactate concentration during the two protocols (GPR vs. No GPR). The analysis of HR showed no significant differences (Figure 2). As can be noted GPR recovery was a significantly higher drop in respiratory rate (Figure 3). This behavior was more pronounced for respiratory rate, reaching a significant value after 6 min of GPR stimulation with respect to baseline $(12.2 \pm 4.15 \mathrm{~min}-1 \mathrm{vs}$. $19.4 \pm 0.4$ min- $1 ; \mathrm{p}<0.001)$ while it increased at the end of the GPR section (13.0 $\pm 1.17 \mathrm{~min}-1$ vs. $15.2 \pm 3.43 \mathrm{~min}-1$; $\mathrm{p}=0.06$ ) to reach a value almost superimposable to baseline. On the opposite during the No GPR recovery the decrease in respiratory rate was more gradual and the final value was still higher with respect to baseline (15.1 $\pm 3.4 \mathrm{~min}-1$ vs. $13.3 \pm 1.34 \mathrm{~min}-1)$. The peripheral oxygen saturation (Figure 4) significantly increased 6 and 13 minutes after peak exercise in both recovery protocols (No GPR: $97.0 \% \pm 0.16 \%$ vs. $96.6 \% \pm 0.81$, $\mathrm{p}<0.001 ; \quad$ GPR: $99.0 \% \pm 0.16 \%$ vs. $96.6 \% \pm 0.77 \%$, $\mathrm{p}<0.001$ ) but in the GPR section subjects reached an average level above the baseline $(97.9 \% \pm 1.60 \%$, $\mathrm{p}<0.001)$ and significantly higher the standard recovery level $(\mathrm{p}<0.001)$. However, the most striking result was the drop in lactate levels after the 13 minutes of GPR recovery period that reached the baseline levels in almost all the volunteers as clearly depicted by Figure 5 (2.4 \pm 0.2 vs. $2.4 \pm 0.3, p=n$.s. with GPR). On the contrary, during the No GPR test the assessment after 13 minutes rest lactate concentration presented no significant changes with respect to the 2 minutes post-stress sampling (15.7 \pm 2.5 vs. $16.2 \pm 2.3$ with No GPR and $2.4 \pm 0.2$ vs. $16.2 \pm 2.4$ with GPR; equivalent to an average decrease of $2.9 \pm 7.6 \%$ vs. $84.5 \pm 3.5 \%, \mathrm{p}<0.001$ ).

\section{Discussion}

This study was aimed at analyzing the effect of a novel approach to proprioceptive stimulation on postexertional recovery after a strenuous exercise in a group of athletes involved in long-lasting maximal aerobic and anaerobic exercise. Recovery under six min of GPR stimulation was associated with an overall improvement in the recorded parameters with a significantly higher decrease in post-exertion respiratory rate, and increase of $\mathrm{SpO}_{2}$. According to these findings, thirteen minutes of GPR stimulation seems to almost completely recover Lactate concentration. Previous studies on the effects of proprioceptive stimulation during/after exercise focused on the devices for WBV. Typically, WBV investigations have been used for short durations, with cumulative exposure of up to $15 \mathrm{~min}$, to enhance exercise level, ${ }^{11}$ and to investigate influences on the central cardiovascular system showing conflicting results. ${ }^{12,13}$ Other studies have reported additional cardiovascular benefits, even in the absence of significant changes in heart rate and blood flow, in the form of the attenuation of the increases in leg arterial stiffness following acute WBV and reduced blood pressure following repeated WBV exercise sessions. ${ }^{14,15}$ However, the reported effects of WBV on peripheral blood flow velocity (BFV) are inconclusive, with some studies indicating an increase in BFV, ${ }^{16-19}$ and others reporting decreases or no change after vibration. $^{20,21}$ Some authors suggested the role of body temperature increase, ${ }^{22}$ while others found no correlation. ${ }^{23}$ Notably, the evaluations of BFV after WBV focused on a specific approach to WBV, in the stand-up position, and on lower limbs circulation. The available studies focusing on the effects of WBV on lactate concentrations aimed at evidencing an increase in energetic demand promoted by WBV during maximal exercise with different results but mainly in favor of this phenomenon thus indicating a possible correlation between lactate concentration, postural stability and balance disorders. ${ }^{24}$ It is worth mentioning that in a previous study, the local vibration applied at the lower limbs, enhanced the expression on Vastus lateralis of dihydrolipoamide dehydrogenase, E3 complex of pyruvate dehydrogenase complex (DLD), the gene coding for the pyruvate dehydrogenase complex able to transform pyruvate into acetyl- coenzyme A. ${ }^{25}$ This result was obtained by analyzing the human muscle fibers after vibrational stimulations for three months. In the actual 
study we observed a significant lactate reduction during WBV recovery with respect to control. Our idea is that the WBV could influence the lactate metabolism and thus produce a faster removal of it. It could be argued that in the noGPR group the lactate removal was slower with respect to many results present in literature describing lactate removal in recovery phase after maximal exertion. ${ }^{9,26}$ However, our result is in accordance with Riganas et al., ${ }^{27}$ who reported a low decrement (15\%) after 10 min of active recovery at $25 \%$ maximal power output following an exhaustive incremental test. When focusing on lactate concentration during post-exercise recovery, the use of WBV was not associated with any improvement in the two available studies on this topic. ${ }^{23,28}$ Several factors can explain the differences between our findings on GPR stimulation and WBV studies: (a) intensity of GPR is controlled to avoid stimulation of profound structures while soliciting the cutaneous mechanoreceptors, (b) GPR is not limited to a single part of the body (vibrational stimulation is usually limited to the legs), (c) the subject is not in a standing position during GPR since he lies in a relaxed supine position transferring all the gravitational energy through the six stimulating parts without compression of the vascular and lymphatic structures to favour circulation. It's very likely that the quick effects on respiratory rate and peripheral oxygenation deserves further investigations to evaluate additional central effects. We are conscious, however, that the present study albeit being randomized and performed in an homogeneous group of subjects, presents one limitation: the sample size of the population is not large and is restricted to a high level trained group of athletes and not also to pathological subjects. We compared GPR to active recovery without any additional tool. These choices can limit generalization and identification of the precise mechanism for the improvement showed by GPR in postexertion recovery. However, the study design and the highly homogenous group of patients significantly enhance the reliability of the obtained results. In conclusion, global proprioceptive resonance is a novel promising approach to proprioceptive stimulation with positive effects on post-exercise recovery by improving rapid restoration of respiratory rate, increased $\mathrm{SpO}^{2}$ and reducing blood lactate concentration. In perspective, the proprioceptive stimulation using body vibrations could have interesting scientific application in the field of rehabilitation.

\section{List of acronyms}

BFV - blood flow velocity

BMI - body mass index

DLD - dihydrolipoamide dehydrogenase

ECG - Electrocardiogram

GPR - Global Proprioceptive Resonance

HR - heart rate

Lac - lactate concentration

$\mathrm{RR}$ - respiratory rate
SBP: sistolic blood pressure

$\mathrm{pO}_{2}$ - peripheral oxygen saturation

WBV - Whole body vibration

\section{Authors contributions}

Each author has contributed to conception, design, and interpretation of data. In particular: FC and PVG selected the recruited subjects and checked their medical aspects. FC, PVG and VV performed the experimental part and collected the data for the subsequent statistical analyses in which also participated. All the authors have contributed substantially to the drafting of the manuscript and to final approval of the version submitted.

\section{Acknowledgments None.}

Funding The work has been completely charged to the research funds provided by FC and PVG

\section{Conflict of Interest}

The authors declare no conflicts of interest.

\section{Ethical Publication Statement}

We confirm that we have read the Journal's position on issues involved in ethical publication and affirm that this report is consistent with those guidelines.

\section{Corresponding Author}

Paola Virginia Gigliotti, MD, Department of Medicine, University of Perugia, Ospedale "Santa Maria della Misericordia”, Laboratory of Sport Physiology, San Candido-Innichen, Italy.

Phone: +39-051349858 Fax: +39-051344859

ORCID iD: 0000-0002-8908-4153

E-mail: pao.gigliotti@gmail.com

E-mails of co-authors

Francesco Coscia: francesco.coscia1@gmail.com ORCID iD: 0000-0003-1900-0302

Alexander Piratinski: apiratinskiy@yandex.ru

ORCID iD: 0000-0002-8611-525X

Tiziana Pietrangelo: tiziana.pietrangelo@unich.it

ORCID iD: 0000-0002-7507-1255

Vittore Verratti: vittore.verratti@unich.it

ORCID iD:0000-0001-8343-9024

Saadsaoud Foued: foued.saadsaoud@univ-msila.dz

ORCID iD: 0000-0001-8228-4497

Igor Diemberger: igor.diemberger@unibo.it

ORCID iD: 0000-0002-3823-3809

Giorgio Fanò-Illic: fanoillic@gmail.com

ORCID iD: 0000-0002-8753-5707

\section{References}

1. Dietz V, Müller R, Colombo G, Locomotor activity in spinal man: significance of afferent input from joint and load receptors. Brain 2002;125:2626-34.

2. Rossignol S, Dubuc R, Gossard JP. Dynamic sensorimotor interactions in locomotion. Physiol Rev 2006;86:89-154. 
3. Lepers R, Knechtle B, Stapley PJ. Trends in Triathlon Performance: Effects of Sex and Age. Sports Med 2013;43:851-63.

4. Proske U, Gandevia SC. The proprioceptive senses: their roles in signaling body shape, body position and movement, and muscle force. Physiol Rev 2012;92:1651-97.

5. Proske U, Allen T. The neural basis of the senses of effort, force and heaviness. Exp Brain Res 2019;237:589-99.

6. Cardinale M1, Wakeling J. Whole body vibration exercise: are vibrations good for you? Br J Sports Med 2005;39:585-9.

7. Lepers R, Knechtle B, Stapley PJ. Larivière C, Trends in Triathlon Performance: Effects of Sex and Age. Sports Med 2013;43:851-63.

8. Larivière C, Gravel D, Arsenault AB, Gagnon D, Loisel P. Muscle recovery from a short fatigue test and consequence on the reliability of EMG indices of fatigue. Eur J Appl Physiol 2003;89:171-7.

9. Devlin J1, Paton B, Poole L, et al. Blood lactate clearance after maximal exercise depends on active recovery intensity. Sports Med Phys Fitness 2014;54:271-8.

10. Pérez EH, Dawood H, Chetty U, et al. Validation of the Accutrend lactate meter for hyperlactatemia screening during antiretroviral therapy in a resource-poor setting. International Journal of Infectious Diseases 2008;12:553-6.

11. Avelar NC, Simão AP, Tossige-Gomes R, et al. Oxygen consumption and heart rate during repeated squatting exercises with or without whole-body vibration in the elderly. J Strength Cond Res 2011;25:3495-500.

12. Rittweger J, Beller G, Felsenberg D. Acute physiological effects of exhaustive whole-body vibration exercise in man. Clin Physiol 2000;20:134-42.

13. Figueroa A, Vicil F, Sanchez-Gonzalez MA. Acute exercise with whole-body vibration decreases wave reflection and leg arterial stiffness. Am J Cardiovasc Dis 2011;1:60-7.

14. Cardinale M, Soiza RL, Leiper JB, et al. Hormonal responses to a single session of whole body vibration exercise in older individuals. Br J Sports Med 2010;44:284-8.

15. Baum K, Votteler T, Schiab J. Efficiency of vibration exercise for glycemic control in type 2 diabetes patients. Int J Med Sci 2007;4:159-63.

16. Otsuki T, Takanami Y, Aoi W, et al. Arterial stiffness acutely decreases after whole-body vibration in humans. Acta Physiol (Oxf) 2008;194:189-94.
17. Cardinale M, Pope MH. The effects of whole body vibration on humans: dangerous or advantageous? Acta Physiol Hung 2003;90:195-206.

18. Lohman EB 3rd, Petrofsky JS, Maloney-Hinds C, et al. The effect of whole body vibration on lower extremity skin blood flow in normal subjects. Med Sci Monit 2007;13:CR71-6.

19. Herrero AJ, Martín J, Martín T, et al. Whole-body vibration alters blood flow velocity and neuromuscular activity in Friedreich's ataxia. Clin Physiol Funct Imaging 2011;31:139-44.

20. Robbins D, Yoganathan P, Goss-Sampson M. The influence of whole body vibration on the central and peripheral cardiovascular system. Clin Physiol Funct Imaging 2014;34:364-9.

21. Button C, Anderson N, Bradford C, et al. The effect of multidirectional mechanical vibration on peripheral circulation of humans. Clin Physiol Funct Imaging 2007;27:211-6.

22. Hazell TJ, Thomas GW, Deguire JR, Lemon PW. Vertical whole-body vibration does not increase cardiovascular stress to static semi-squat exercise. Eur J Appl Physiol 2008;104:903-8.

23. Edge J, Mündel T, Weir K, Cochrane DJ. The effects of acute whole body vibration as a recovery modality following high-intensity interval training in well-trained, middle-aged runners. Eur J Appl Physiol 2009;105:421-8.

24. Robbins D1, Yoganathan P, Goss-Sampson M. The influence of whole body vibration on the central and peripheral cardiovascular system. Clin Physiol Funct Imaging 2014;34:364-9.

25. Pietrangelo T, Mancinelli R, Toniolo L, et al. Effects of local vibrations on skeletal muscle trophism in elderly people: mechanical, cellular, and molecular events. Int J Mol Med 2009;24:50312

26. Larivière C, Gravel D, Arsenault AB, et al. Muscle recovery from a short fatigue test and consequence on the reliability of EMG indices of fatigue. Eur $\mathrm{J}$ Appl Physiol 2003;89:171-6.

27. Riganas CS, Papadopoulou Z, Psichas N, et al. The rate of lactate removal after maximal exercise: the effect of intensity during active recovery. J Sports Med Phys Fitness 2015;55:1058-6.

28. Manimmanakorn N, Ross JJ, Manimmanakorn A, Lucas SJ, Hamlin MJ. Effect of whole-body vibration therapy on performance recovery. Int $\mathrm{J}$ Sports Physiol Perform. 2015 Apr;10(3):388-95

Submitted: November 9, 2020 Accepted for publication November 9, 2020 